\title{
THE TOOTH BRUSH AND METHODS OF CLEANING THE TEETH.
}

\author{
A Report to the National Dental Association by \\ Jules J. Sarrazin, President, the American Academy of Periodontology, \\ New Orleans, La., Reporter.
}

(Read before a General Session of the National Dental Association at Its Twenty-third Annual Meeting, New Orleans, La., October 20-24, 1919.)

A

COMMITTEE has undertaken the preparation of this report in response to the Academy's request that it present a description of a tooth brush of approved design and a method of brushing the teeth which will prove of service to the general practitioner of dentistry in instructing patients in the personal care of the teeth.

The committee has secured from the members of its Council opinions resulting from their clinical experiences, and has based its report upon such collective views, after also embodying those resulting from a general meeting of the membership of The American Academy of Periodontology.

The effort has been to attempt the standardization of the personal care of the mouth by recommending a brush of an accepted design, which may be generally adopted, and an efficient method of brushing the gums and teeth which may be universally applied. Both brush and method have been demonstrated by members of the Academy to be efficacious and non-injurious.

In recommending one form of tooth brush and one method of brushing the teeth, the Academy does not wish it understood that it discredits all brushes of dissimilar design or all methods of brushing the teeth which do not conform to the method outlined, but, that it endorses a brush and a method offering the greatest advantages.

The opinion has been unanimously expressed that a brush larger than one and one-half inches in length in the brush head, and what is known as the crosswise or mesio-distal stroke method, are both injurious and inefficient.

With this statement of its objects the report is presented to the National Dental Association with a request for iis adoption.

\section{The Tooth Brush.}

\section{For Adults:}

Brush Head.

Total length of brush head, 11/4".

Number of bristle tufts in row at neck, at most, 3 .

Number of bristle tufts in row at extremity, 2.

Space between rows, $3 / 32^{\prime \prime}$ to $1 / 8$ ".

Length of bristle tufts at neck, $3 / 8^{\prime \prime}$ to $1 / 2^{\prime \prime}$.

Length of bristle tufts at end, $1 / 4$ " to $3 / 8$ ".

Bristles in tufts should be rounded. The brushing surface should present a slanting straight line from the neck to the end of the brush head, and it should be convex from side to side.

Bristles should be stiff. 


\section{Handle.}

Should be fairly rigid.

Should be 6 inches (entire length of handle and brush head.)

The neck should be long and slender.

For Children over three years of age:

Brush Head.

Total Length, 1".

Number of bristle tufts at neck, 2 .

Number of bristle tufts at extremity, 1.

Otherwise, with the exception of a general decrease in size, the child's brush should resemble the adult brush.

For Children under three years of age: Brush Head.

Total length, $7 / 8^{\prime \prime}$.

Single bristle tufts set lengthwise of brush head, 5 .

Length of bristle tufts at neck, $5 / 16^{\prime \prime}$.

Length of bristle tufts at extremity, $3 / 16^{\prime \prime}$.

Otherwise, with the exception of a general decrease in size, it should resemble the first child's brush described.

Methods of Brushing the Teeth.

The American Academy of Periodontology recognizes four effective methods of brushing the teeth, known as:

1. The rolling stroke method.

2. The up and down stroke method.

3. The circulat stroke method.

4. The vibratory method.

METHOD (1). The rolling stroke method, which takes precedence over other methods, consists of placing the brush in the mouth, bristle ends $u p$, for the upper teeth, and down for the lower teeth, and rolling the brush toward the gums, sweeping the bristle over the gums and teeth to their occlusal surfaces.

This motion brushes the buccal and labial surfaces of all the teeth above and below, and the lingual surfaces of the molars and bicuspids in both arches.

The lingual surfaces of the incisors and cuspids are brushed by placing the brush parallel to the tongue, with the bristle ends on the roof of the mouth for the upper teeth and on the floor of the mouth for the lower teeth, drawing the brush forward over the gums and teeth to their incisal edges.

The upper and lower jaws are brushed separately.

The occlusal surfaces are brushed with a forward and backward, and side to side stroke of the brush.

MethoD (2). The up and down stroke method is presented as a practicable compromise between the rolling and the circular stroke methods, as one whose technic can be readily mastered by many whose personal mouth care might otherwise be less efficient. It consists of placing the brush with the bristle ends toward the gums and while in that position of carrying the brush down over the gums and teeth to their occlusal surfaces, and up again for the upper teeth, and the reverse for the lower teeth.

The buccal and labial surfaces of the upper and lower jaws may be brushed separately, or the teeth may be placed edge to edge and both upper and lower jaws brushed at the same time.

The lingual surfaces are brushed 10 the same way.

The occlusal surfaces are brushed with an in and out, and side to side stroke of the brush.

METHoD (3). The circular stroke method consists of placing the brush with the bristle ends toward the gums, and with a circular motion brushing the gums and teeth. The buccal and labia] surfaces of each jaw may be brushed separately, or the teeth may be placed 
edge to edge, and both upper and lower jaws brushed at the same time.

The lingual and occlusal surfaces are brushed with the same circular stroke.

METHOD (4). The "vibratory meth$o d$ " of brushing the teeth consists of placing the brush horizontal to the long axes of the teeth with the bristle ends resting on the tooth surfaces. When pressure is applied to the brush and a very short mesio-distal stroke executed, the bristle ends enter the embrasures between the teeth covered by the brush. The stroke, if it may be called a stroke, is so short that it does not carry the bristles beyond the teeth upon which they rested before the stroke was begun.

In this manner all the surfaces of the teeth are brushed.

Methon 1 is a simple and effective method favored by the great majority of the members of the American Academy of Periodontology.

The gums and teeth should be brushed before breakfast and after meals. The most important time to brush is at night.

Those portions of the individual mouth which demand the most care should be brushed first. In general, the following rotation may be followed:

\section{Lower jaw.}

Lingual surfaces of the gums and teeth.

Buccal surfaces of the gums and teeth.

Labial surfaces of the gums and teeth. Upper jaw.

Buccal surfaces of the gums and teeth.

Labial surfaces of the gums and teeth.

Lingual surfaces of the gums and teeth.

Occlusal surfaces of both the upper and lower teeth.

The brush should be rinsed frequently in clear water during the brushing.

The tongue should be brushed in the morning.
The brush should be thoroly rinsed after its use, and be placed where it will dry.

FLoss.

Waxed ribbon floss should be used after brushing the teeth and gums. The majority of the members of the Academy recommend its use at night. It should be carefully rubbed over the proximal surfaces of the teeth and should not be allowed to injure the gums. The distance between the finger ends, when the floss is used, should be at all times reduced to the minimum, approximately one-half to three quarters of an inch. The floss should be stretched taut over the ends of the fingers, or finger and thumb, not wound around them.

Special care must be given to the proximal surfaces of teeth bounding spaces where one or more teeth are missing. These surfaces may be brushed with a single row bristle brush, where the space permits, or they may be cleansed with ribbon floss.

Ribbon floss threaded on a bodkin and passed between the dummy teeth and the soft tissues should be used to cleanse the under surfaces of fixed bridges and the abutment teeth.

INSTRUCTIONS INTENDED FOR THE LAYMAN.

\section{By THe Clock.}

Brush your teeth for 5 minutes, 3 or four times each day.

1. Before breakfast.

2. After breakfast.

3. After luncheon.

4. Before going to bed.

Use a small brush.

Brush the gums and teeth.

Be sure to reach both sides of the rearmost molars.

\section{To BRUsh the Teeth.}

Place the tooth brush, ends of the bristles $u p$, for the upper teeth, between the cheek and the gums; turn the brush 
toward the gums, sweeping the bristles down over the gums and teeth to the cutting edges of the teeth. Reverse for the lower teeth and brush $u p$.

This motion brushes the cheek side of all teeth and the tongue side of the back teeth.

Brush the inside of the front teeth by putting the brush, bristles up, on the roof of the mouth for the upper teeth, and on the floor of the mouth for the lower teeth, pulling the brush outward over the gums and teeth.

Brush the grinding surfaces of the teeth with an in and out and side to side stroke of the brush.

Rinse the brush in clear water frequently while brushing the teeth and hang it up to dry when not in use.

A tooth powder, paste or a solution of common table salt may be used as a dentrifice once each day. Use clear water at other times.

Special Committee.

Gillette Hayden, D. D. S., Chairman, 289 E. State St., Columbus, Ohio.
Austin F. James, D. D. S., 1615 Marshall Field Annex, Chicago, Ill.

Andrew J. McDonagh, L. D. S., 2 Bloor St., East, Toronto, Canada.

Council of the American ACademy of Peridontology.

J. Herbert Hood, D. D. S., Chairman, 1101 Guardian Bldg., Cleveland, Ohio.

Clyde M. Gearhart, D. D. S., 1624 I St., Washington, D. C.

Paul R. Stillman, D. D. S., 52 Vanderbilt Ave., New York City, N. Y.

John Oppie McCall, A. B., D. D. S., 437 Franklin St., Buffalo, N. Y.

R. R. Johnston, D. D. S., Westinghouse Bldg., Pittsburgh, Pa.

Jules J. Sarrazin, D. D. S., President, 422-424 Godchaux Bldg., New Orleans, La.

Andrew J. McDonagh, L. D. S., President-elect, 2 Bloor St., East, Toronto, Canada.

Grace Rogers Spalding, D. D. S., Secretary-Treasurer, 75 Eliot St., Detroit, Mich. 